\title{
Nedd8 processing enzymes in Schizosaccharomyces pombe
}

\author{
Jean E O'Donoghue ${ }^{1}$, Dawadschargal Bech-Otschir ${ }^{1}$, Ida B Larsen² ${ }^{2}$ Mairi Wallace ${ }^{1}$, Rasmus Hartmann-Petersen ${ }^{2}$ \\ and Colin Gordon ${ }^{1 *}$
}

\begin{abstract}
Background: Conjugation of the ubiquitin-like modifier Nedd8 to cullins is critical for the function of SCF-type ubiquitin ligases and thus facilitates ubiquitin conjugation and ultimately degradation of SCF substrates, including several cell cycle regulators. Like ubiquitin, Nedd8 is produced as a precursor that must first be processed before it becomes active. In Saccharomyces cerevisiae this is carried out exclusively by the enzyme Yuh1.

Results: Here we show that in the fission yeast, Schizosaccharomyces pombe, the Yuh1 orthologue, Uch1, is not the sole Nedd8 processing enzyme. Instead it appears that deubiquitylating enzymes can efficiently process the Nedd8 precursor in vivo.
\end{abstract}

Conclusions: Several enzymes contribute to Nedd8 precursor processing including a number of deubiquitylating enzymes.

Keywords: Ubiquitin, Nedd8, Rub1, Cullin, Protein degradation, Precursor processing

\section{Background}

Post translational modification of proteins with ubiquitin and ubiquitin-like modifiers is an essential mechanism that regulates many cellular processes including protein degradation, endocytosis, DNA repair and cell signalling. The protein called Nedd8 in humans and Rub1 in budding yeast is a ubiquitin-like modifier that is covalently conjugated to a lysine residue in the cullin subunits of SCF-type E3 ubiquitin-protein ligases [1] and possibly a few other proteins [2,3]. The Nedd8 modification, also known as neddylation, of cullins is important for function and the recruitment of E2s and other components [4-6] to SCF-type ubiquitin ligases and therefore facilitates ubiquitin conjugation of SCF substrates. Similar to ubiquitin, Nedd8 conjugation requires an E1, E2 and E3 enzyme [3]. In the fission yeast Schizosaccharomyces pombe [6,7], as in mammals [8], the null mutation of either Nedd8 itself or components of its conjugation pathway, i.e. its E1 or E2 enzymes, are lethal to the cell. Curiously, the Nedd8 system is not essential for viability of the budding yeast Saccharomyces cerevisiae [9,10].

\footnotetext{
* Correspondence: colin.gordon@hgu.mrc.ac.uk

'MRC Human Genetics Unit, Western General Hospital, Crewe Road, Edinburgh EH4 2XU, UK

Full list of author information is available at the end of the article
}

The reason for this difference is unknown, but could reflect differences in Nedd8 substrates between these organisms.

Just as ubiquitylation is reversed by ubiquitin isopeptidases, Nedd8 conjugation is reversed by Nedd8 isopeptidases in a process known as deneddylation. The best characterized deneddylating enzymes include the COP9 signalosome subunit Csn5 [11-13] and NEDP1 (also known as SENP8 and DEN1) $[14,15]$. In general these enzymes are surprisingly specific for Nedd8 over ubiquitin $[3,14]$, but some have been shown to have dual specificity $[15,16]$. More recently, certain cysteine proteases, encoded by herpesviruses, were also shown to display deneddylase activity [17].

In all eukaryotic organisms the NEDD8 gene is conserved (Additional file 1: Figure S1) and encodes a non-conjugatable precursor that contains one or more residues downstream of the mature $\mathrm{C}$-terminus at position 76. To generate mature Nedd8 from the inactive precursor, specific hydrolases cleave off the $\mathrm{C}$-terminal residues generating a diglycine motif in the mature Nedd8 C-terminus [1]. This precursor processing is strictly required for Nedd8's recognition by the E1 enzyme and so the enzymes that catalyse this processing must be essential for Nedd8 function.
C Biomed Central

(c) 2013 O'Donoghue et al.; licensee BioMed Central Ltd. This is an Open Access article distributed under the terms of the Creative Commons Attribution License (http://creativecommons.org/licenses/by/2.0), which permits unrestricted use, distribution, and reproduction in any medium, provided the original work is properly cited. 
In $S$. cerevisiae the sole Nedd8-precursor processing enzyme has been identified as Yuh1 [18] (S. pombe Uch1, Additional file 1: Figure S2). In the Yuh1 null mutant, there is no longer any neddylation of cullin1 [18]. Here, we present results aimed at identifying the Nedd8 processing enzyme(s) important for Nedd8 function in $S$. pombe. We show that unlike the situation in budding yeast, $S$. pombe cells contain several enzymes capable of processing the Nedd 8 precursor.

\section{Methods}

\section{S. pombe strains and techniques}

Fission yeast strains used in this study are derivatives of the wild type heterothallic strains $972 h^{-}$and $975 h^{+}$. Standard genetic methods and media were used and $S$. pombe transformations were performed using the lithium acetate procedure [19]. The PCR mutagenesis was performed according to a previously published procedure [20].

\section{Antibodies}

The antibody to tubulin was the TAT-1 monoclonal (Sigma). The antibodies to $S$. pombe Cul1/Pcu1 have been described before [21], and were kindly provided by Prof. Dieter A. Wolf (La Jolla, USA).

\section{Plasmids, expression and purification}

The E. coli expression constructs used here were wild type cDNA encoding Uch1, Uch2, Nep1 and Nep2 subcloned to the pGEX-KG or pGEX-6p-1 vector (GE Healthcare). All recombinant proteins were expressed as glutathione S-transferase (GST) fusion proteins in E. coli BL21 (DE3) pLysS and purified on glutathioneSepharose beads (GE Healthcare) by standard methods. The protein: bead ratio was normalized by SDS-PAGE and Coomassie staining.

\section{Assays}

For analyses of the deneddylating activity of purified recombinant proteins and in cell extracts, the flourogenic substrate, Nedd8-7-amino-4-methylcoumarin (Nedd8AMC) (Boston Biochemicals) was used according the instructions provided by the manufacturer and as described previously [22]. Protein concentrations were determined by Bradford assays (Pierce). Ubiquitin aldehyde (Enzo Life Sciences) was used at $5 \mu \mathrm{M}$, while o-phenanthroline (Sigma) was used at $5 \mathrm{mM}$.

\section{Results}

\section{Uch1/Yuh1 is a deneddylating enzyme}

The orthologue of YUH1 in S. pombe is $u c h 1^{+}$, which has a paralogue, $u c h 2^{+}$. To investigate the role of $u c h 1^{+}$ in the Nedd8 pathway, we created a null mutation in the $u c h 1^{+}$gene by PCR mutagenesis.
We first noted that cells carrying this $\Delta u c h 1:: G 418$ mutation were viable (Figure 1) and appeared to have no physiological ill effects. If Uch1, as Yuh1 in S. cerevisiae, was the sole Nedd8-precursor processing enzyme, we would have expected the $u c h 1^{+}$deletion to be lethal. In order to detect any change in the neddylation status of a Nedd8 substrate we replicated the experiment carried out in S. cerevisiae [18]. We used an antibody to fission yeast cullin 1 (Cul1/Pcu1) [21] to detect Cul1 in cell extracts prepared from the $\Delta u c h 1$ strain. Unlike in S. cerevisiae, Cul1 was neddylated to a similar extent in wild type and $\Delta u c h 1 S$. pombe strains (Figure 2).

To assess whether or not $S$. pombe Uch1 could process Nedd8-precursor in vitro we purified a GST-tagged version of Uch1 and used Nedd8-AMC as a substrate. From this, we observed that Uch1 was indeed capable of processing Nedd8-AMC in vitro (Figure 3).

\section{Uch2/Uch37 accounts for some deneddylating activity in fission yeast}

In an attempt to isolate other potential Nedd8-precursor processing enzymes we turned to $u c h 2^{+}$, a paralogue of $u c h 1^{+}$, and carried out the same set of experiments as described above using a GST-tagged version of Uch2 (Figure 3), a $\Delta u c h 2:: u r a 4$ strain, and a double $\Delta u c h 1 \Delta u c h 2$ strain (Figures 1-2). As can be seen from these data, despite the fact that Uch2 is capable of processing Nedd8AMC in vitro (Figure 3), the deletion of both homologues of YUH1 in S. pombe does not result in any loss of Cul1 neddylation (Figure 2). In addition, no loss in cell viability was apparent (Figure 1), as would be expected in a Nedd8 compromised S. pombe strain.

\section{Deneddylating enzymes are functionally redundant}

Two genes that appear to be orthologues of the mammalian NEDP1/DEN1 have been identified in $S$. pombe [23]. These are $n e p 1^{+}$and $n e p 2^{+}$. In mammalian cells NEDP1/DEN1 has been described as a Nedd8-precursor processing enzyme [24], and while $S$. pombe has two such orthologues, $S$. cerevisiae has none. We hypothesised therefore that these enzymes may possess the remaining Nedd8-precursor processing activity in $S$. pombe that was unaccounted for by the two YUH1 orthologues. To ascertain this, we created GST-tagged versions of Nep1 and Nep2 and showed that these have processing activity in vitro (Figure 3). We then created null mutants in each of these genes, $\Delta$ nep $1:: G 418$ and $\Delta$ nep2::arg3, and crossed them to our double mutant $\Delta u c h 1 \Delta u c h 2$ strain in attempt to completely abrogate processing function in fission yeast. Surprisingly, we found that this $\Delta n e p 1 \Delta n e p 2 \Delta u c h 1 \Delta u c h 2$ strain was entirely viable (Figure 1), and Cul1 could be efficiently neddylated in this strain (Figure 2). 


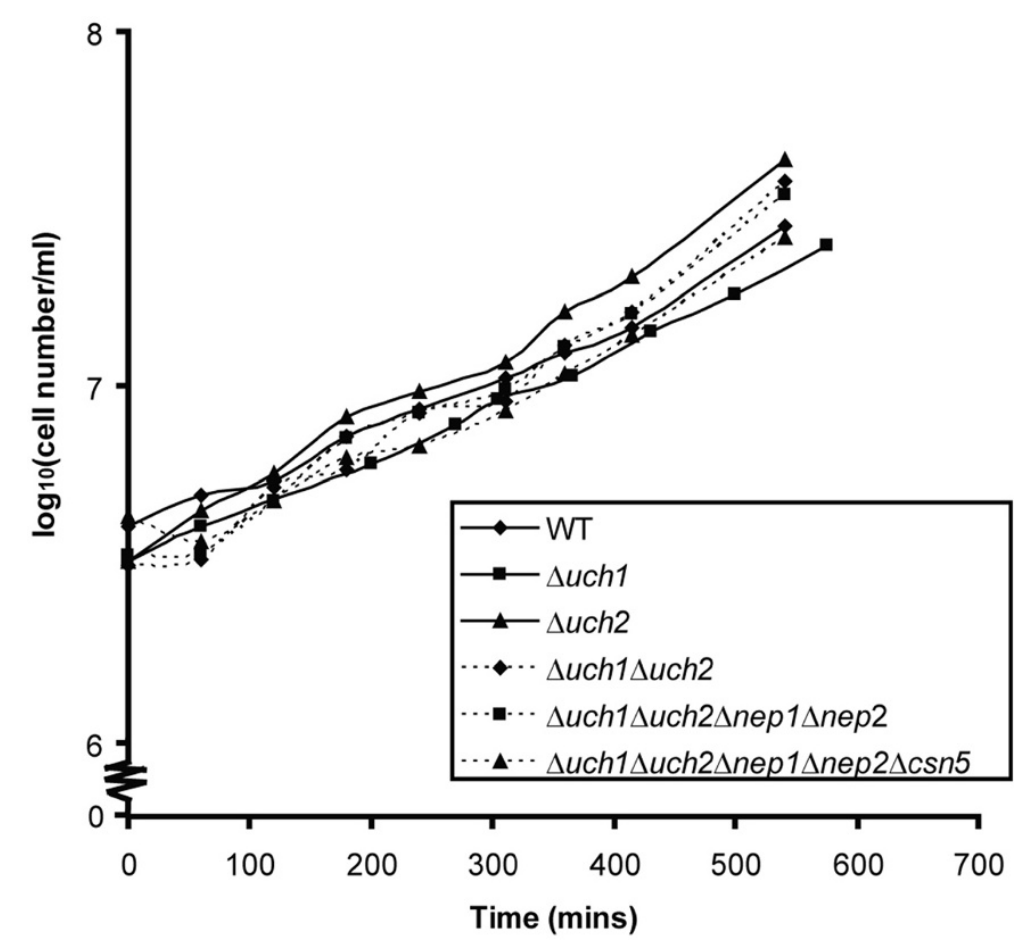

Figure 1 Growth curves of wild type (WT) and mutant strains. All mutant strains were viable and showed growth characteristics similar to the WT strain. The doubling time was about 2 hours and 45 minutes.

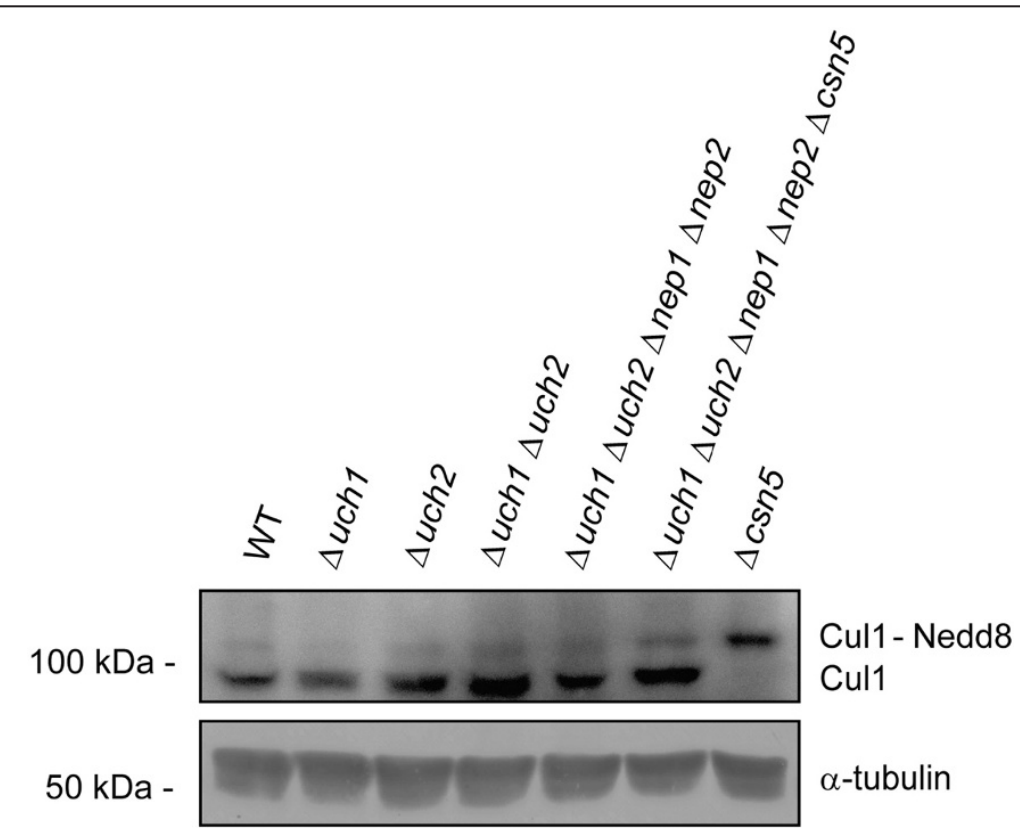

Figure 2 SDS-PAGE and western blotting showing the neddylation status of Cul1 in the wild type (WT) and mutant strains. In all strains a band corresponding to a neddylated Cul1 can be detected using an anti-Cul1 antibody. Tubulin was used to check for equal loading. 


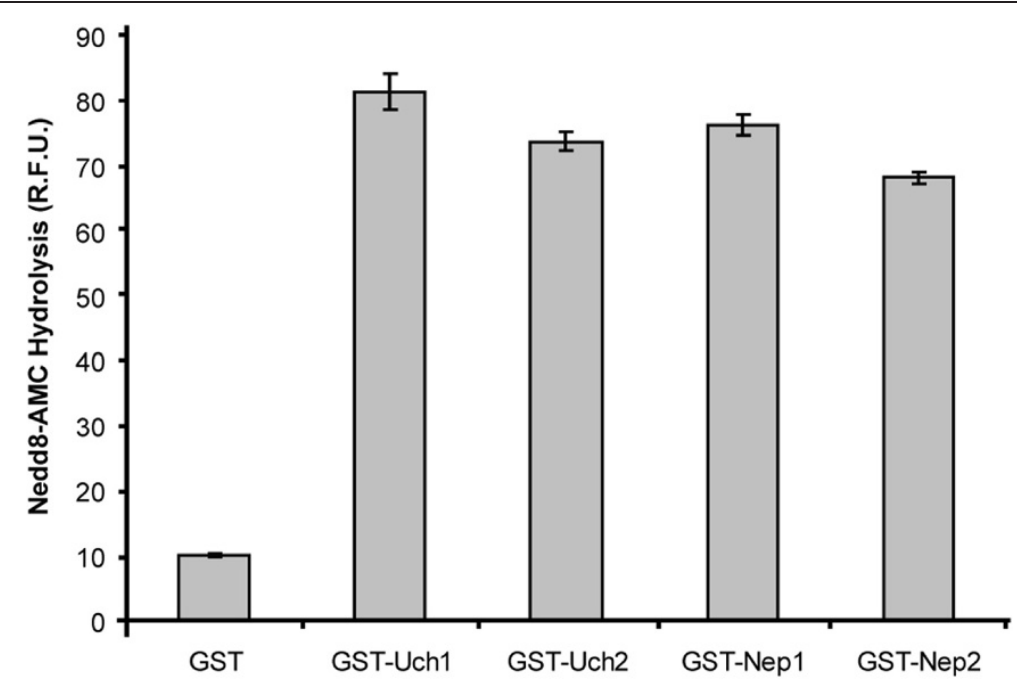

Figure 3 Nedd8-AMC processing activity of Uch1, Uch2, Nep1 and Nep2. While incubation with GST alone does not result in cleavage of Nedd8-AMC, GST-Uch1, GST-Uch2, GST-Nep1 and GST-Nep2 can all cleave Nedd8-AMC ( $n=5$, S.E.M. shown as error bars).

The final candidate we examined as a potential Nedd8 processing enzyme in fission yeast was $c s n 5^{+}$. This subunit of the COP9/signalosome complex is well described as a metalloprotease capable of deconjugating Nedd8 from its cullin substrates [25,26]. Indeed, as observed before $[21,26]$, hyperneddylation of Nedd8 was readily apparent in our $\Delta \operatorname{csn} 5$ ::ura4 mutant (Figure 2). Whether Csn5 can process Nedd8-precursor, however, is unclear. The $\Delta \operatorname{csn} 5$ mutant was crossed to the quadruple knockout strain to create a $\Delta n e p 1 \Delta n e p 2 \Delta u c h 1 \Delta u c h 2 \Delta \operatorname{csn} 5$ strain. Even this quintuple knockout strain was viable (Figure 1). However, the hyperneddylation of Cul1 observed in the $\Delta \operatorname{csn} 5$ single mutant (Figure 2) was not as apparent in the $\Delta n e p 1 \Delta n e p 2 \Delta u c h 1 \Delta u c h 2 \Delta \operatorname{csn} 5$ strain, suggesting that a reduced amount of processed Nedd8 is available for Cul1 modification in this background (Figure 2). Despite this effect on Cul1 neddylation, the quintuple knockout strain did not display any growth defect when compared to the wild type control or the other mutants (Figure 1).

\section{Deubiquitylating enzymes also provide deneddylating activity}

In an attempt to estimate the remaining Nedd8precursor processing activity we examined the Nedd8AMC processing activity of the $\Delta n e p 1 \Delta n e p 2 \Delta u c h 1 \Delta u c h 2$ and $\Delta$ nep $1 \Delta n e p 2 \Delta u c h 1 \Delta u c h 2 \Delta c s n 5$ strains. This was done by preparing protein extracts of the strains and incubating with Nedd8-AMC as described previously [22]. The results revealed that albeit the processing activity in these strains was reduced, it was by no means eliminated (Figure 4). Most deubiquitylating and deneddylating enzymes in fission yeast are cysteine proteases, but a few are metalloproteases [27]. To determine what may be responsible for this remaining activity in the quintuple mutant we used two enzymatic inhibitors, ubiquitin aldehyde and o-phenanthroline. Ubiquitin aldehyde is used to inhibit deubiquitylating enzymes, while o-phenanthroline inhibits metalloproteases. As evident from the data, while ubiquitin aldehyde can inhibit some of the remaining activity from the $\Delta$ nep $1 \Delta$ nep $2 \Delta u \operatorname{ch} 1 \Delta u \operatorname{ch} 2 \Delta \operatorname{csn} 5$ strain, o-phenanthroline cannot (Figure 4). This implies that the remaining activity present in the $\Delta n e p 1 \Delta n e p 2 \Delta u c h 1$ $\Delta u \operatorname{ch} 2 \Delta \operatorname{csn} 5$ strain is due to the activity of ubiquitin protease(s), but not metalloproteases.

\section{Discussion}

In the present work we found that unlike in S. cerevisiae, the Nedd8-precursor processing activity in S. pombe is not solely associated with the YUH1 orthologue $u c h 1^{+}$. In fact the deletion of five candidate genes for Nedd8precursor processing activity appears to have little physiological effect on the cell, despite the predicted lethality associated with the lack of Nedd8-precursor processing in fission yeast.

At first we were surprised by these results, but perhaps it is not so unexpected given what we know of Nedd8 in S. pombe, i.e. that lack of Nedd8-precursor processing will undoubtedly lead to death of the cell, and that Nedd 8 and ubiquitin are $50 \%$ identical in amino acid sequence. Perhaps the requirement for Nedd8-precursor processing, forces the cell to utilize those enzymes which perform a similar function for ubiquitin, turning them into Nedd8-precursor processing enzymes. This is suggested by the remaining processing activity and cell viability once the genes for the five enzymes Uch1, Uch2, Nep1, Nep2 and Csn5 were deleted. Despite these data, it is possible that in the wild type cell under normal 


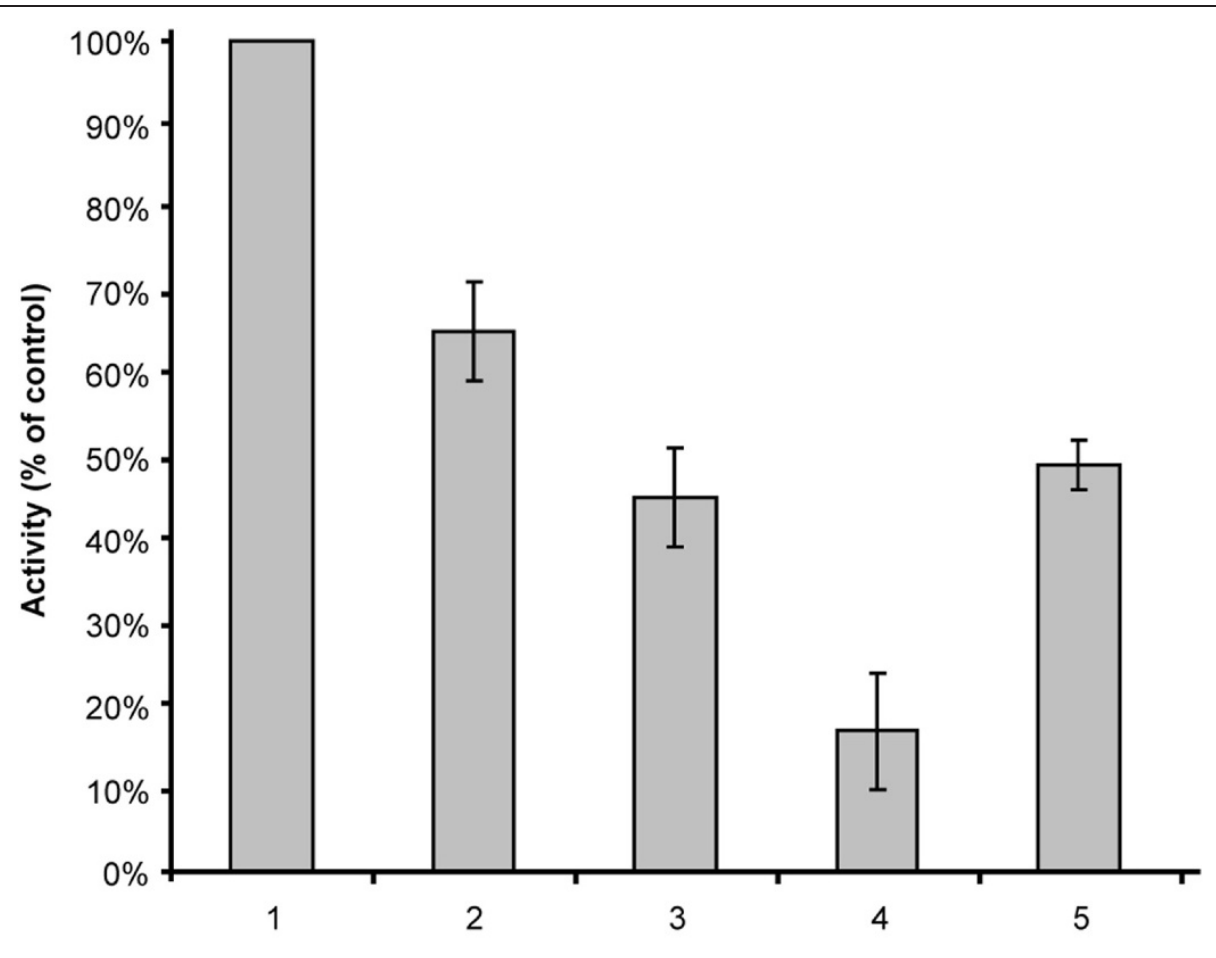

1. WT

2. $\Delta u c h 1 \Delta u c h 2 \Delta$ nep1 1 nep2

3. $\Delta u c h 1 \Delta u c h 2 \Delta n e p 1 \Delta n e p 2 \Delta c s n 5$

4. $\Delta u c h 1 \Delta u c h 2 \Delta n e p 1 \Delta n e p 2 \Delta c s n 5+$ ubiquitin aldehyde

5. $\Delta u c h 1 \Delta u c h 2 \Delta n e p 1 \Delta$ nep $2 \Delta c s n 5+$ o-phenanthroline

Figure 4 Nedd8-AMC processing ability of some of the mutant strains. The $\Delta$ uch $1 \Delta$ uch $2 \Delta$ nep $1 \Delta$ nep 2 strain shows reduced levels of activity ( $66 \%$ of wild type) while the 5 gene deletion strain, $\Delta$ uch $1 \Delta$ uch $2 \Delta$ nep $1 \Delta$ nep $2 \Delta \operatorname{cs} n 5$ shows even less activity ( $45 \%$ of wild type). However there is still processing activity unaccounted for by these 5 enzymes. This activity can be further reduced upon incubation with ubiquitin-aldehyde, but not o-phenanthroline. This suggests that other ubiquitin proteases could account for the remaining activity, but that these proteases are not metalloproteases. ( $n=5$, S.E.M. shown as error bars).

physiological conditions, there is only one main Nedd8precursor processing enzyme in $S$. pombe, and it is only when the function of this enzyme is lost that the cell recruits other enzymes to perform the vital task of Nedd8precursor processing in fission yeast.

The observation that hyper neddylation of Cul1 is lost in the quintuple mutant also suggests that these five enzymes provide the cell with most of the Nedd8precursor processing activity. However, since the quintuple mutant is viable, a limiting amount of processed Nedd8 must be sufficient to support neddylation.

Some DUBs are regulated on the level of their subcellular localization [28]. We note that our deneddylation assays were performed in cell extracts, and therefore do not take this into account.

Recently, Nedd8 overexpression was found to result in neddylation of ubiquitin substrates catalyzed by ubiquitin enzymes $[29,30]$. This atypical neddylation suggests that ubiquitin activating enzymes can moonlight as Nedd8 activating enzymes. The results presented here suggest that deubiquitylating enzymes similarly may also target Nedd8. These results are also in agreement with a recent paper on budding yeast Nedd8 (Rub1) cleavage [31], which shows that heterologous Rub1-ubiquitin chains are disassembled by the COP9 signalosome and proteasome-associated DUBs. Hence, although Yuh1 in budding yeast appears to be the major processing enzyme in Saccharomyces cerevisiae, the profound structural similarity between ubiquitin and Nedd8 [31] leads to functional cross-talk in both yeasts.

\section{Conclusions}

Unlike the situation in budding yeast, several enzymes contribute to Nedd8 precursor processing in S. pombe, 
including a number of deubiquitylating enzymes. This functional redundancy may be connected with the essential nature of Nedd 8 conjugation in fission yeast.

\section{Additional file}

\section{Additional file 1: Supporting Information.}

\section{Abbreviations}

AMC: 7-amino-4-methylcoumarin; GST: Glutathione S-transferase;

S.E.M.: Standard error of mean; WT: Wild type.

\section{Competing interests}

The authors declare that they have no competing interests.

\section{Authors' contributions}

JOD, DBO, IBL and MW carried out experiments. CG and RHP conceived the study. JOD and RHP drafted the manuscript. All authors have read and approved the final manuscript.

\section{Acknowledgements}

We thank Prof. Dieter A. Wolf for providing the Cul1 antibody. We thank Dr Klavs B. Hendil for helpful discussion and comments on the manuscript. This work has been supported financially by grants to RHP and CG from the Medical Research Council, the Lundbeck Foundation and the Danish Natural Science Research Council. The funding sources had no role in study design, data collection, data presentation or in writing of the manuscript.

\section{Author details}

${ }^{1}$ MRC Human Genetics Unit, Western General Hospital, Crewe Road, Edinburgh EH4 2XU, UK. ²Department of Biology, University of Copenhagen, Ole Maaløes Vej 5, DK-2200, Copenhagen, Denmark.

\section{Received: 7 August 2012 Accepted: 8 March 2013}

Published: 15 March 2013

\section{References}

1. Kamitani T, Kito K, Nguyen HP, Yeh ET: Characterization of NEDD8, a developmentally down-regulated ubiquitin-like protein. J Biol Chem 1997, 272:28557-28562.

2. Xirodimas DP, Sundqvist A, Nakamura A, Shen L, Botting C, Hay RT: Ribosomal proteins are targets for the NEDD8 pathway. EMBO Rep 2008, 9:280-286.

3. Rabut G, Peter M: Function and regulation of protein neddylation. 'Protein modifications: beyond the usual suspects' review series. EMBO Rep 2008, 9:969-976.

4. Besten W, Verma R, Kleiger G, Oania RS, Deshaies RJ: NEDD8 links cullinRING ubiquitin ligase function to the p97 pathway. Nat Struct Mol Biol 2012, 19:511-516.

5. Bandau S, Knebel A, Gage ZO, Wood NT, Alexandru G: UBXN7 docks on neddylated cullin complexes using its UIM motif and causes HIF1alpha accumulation. BMC Biol 2012, 10:36.

6. Osaka F, Saeki M, Katayama S, Aida N, Toh E, Kominami K, Toda T, Suzuki T, Chiba T, Tanaka K, Kato S: Covalent modifier NEDD8 is essential for SCF ubiquitin-ligase in fission yeast. EMBO J 2000, 19:3475-3484.

7. Girdwood D, Xirodimas DP, Gordon C: The essential functions of NEDD8 are mediated via distinct surface regions, and not by polyneddylation in Schizosaccharomyces pombe. PLoS One 2011, 6:e20089.

8. Tateishi K, Omata M, Tanaka K, Chiba T: The NEDD8 system is essential for cell cycle progression and morphogenetic pathway in mice. J Cell Biol 2001, 155:571-579.

9. Lammer D, Mathias N, Laplaza JM, Jiang W, Liu Y, Callis J, Goebl M, Estelle M: Modification of yeast Cdc53p by the ubiquitin-related protein rub1 $\mathrm{p}$ affects function of the SCFCdc4 complex. Genes Dev 1998, 12:914-926.

10. Liakopoulos D, Doenges G, Matuschewski K, Jentsch S: A novel protein modification pathway related to the ubiquitin system. EMBO J 1998 17:2208-2214
11. Schwechheimer C: The COP9 signalosome (CSN): an evolutionary conserved proteolysis regulator in eukaryotic development. Biochim Biophys Acta 2004, 1695:45-54.

12. Cope GA, Deshaies RJ: COP9 signalosome: a multifunctional regulator of SCF and other cullin-based ubiquitin ligases. Cell 2003, 114:663-671.

13. Schmaler T, Dubiel W: Control of Deneddylation by the COP9 Signalosome. Subcell Biochem 2010, 54:57-68.

14. Shen LN, Liu H, Dong C, Xirodimas D, Naismith JH, Hay RT: Structural basis of NEDD8 ubiquitin discrimination by the deNEDDylating enzyme NEDP1. EMBO J 2005, 24:1341-1351.

15. Gan-Erdene T, Nagamalleswari K, Yin L, Wu K, Pan ZQ, Wilkinson KD: Identification and characterization of DEN1, a deneddylase of the ULP family. J Biol Chem 2003, 278:28892-28900.

16. Hemelaar J, Borodovsky A, Kessler BM, Reverter D, Cook J, Kolli N, Gan-Erdene T, Wilkinson KD, Gill G, Lima CD, Ploegh HL, Ovaa H: Specific and covalent targeting of conjugating and deconjugating enzymes of ubiquitin-like proteins. Mol Cell Biol 2004, 24:84-95.

17. Gastaldello S, Hildebrand S, Faridani O, Callegari S, Palmkvist M, Di GC, Masucci MG: A deneddylase encoded by Epstein-Barr virus promotes viral DNA replication by regulating the activity of cullin-RING ligases. Nat Cell Biol 2010, 12:351-361.

18. Linghu B, Callis J, Goebl MG: Rub1p processing by Yuh1p is required for wild-type levels of Rub1p conjugation to Cdc53p. Eukaryot Cell 2002, 1:491-494.

19. Moreno S, Klar A, Nurse P: Molecular genetic analysis of fission yeast Schizosaccharomyces pombe. Methods Enzymol 1991, 194:795-823.

20. Bahler J, Wu JQ, Longtine MS, Shah NG, McKenzie A III, Steever AB, Wach A, Philippsen P, Pringle JR: Heterologous modules for efficient and versatile PCR-based gene targeting in Schizosaccharomyces pombe. Yeast 1998, 14:943-951.

21. Schmidt MW, McQuary PR, Wee S, Hofmann K, Wolf DA: F-box-directed CRL complex assembly and regulation by the CSN and CAND1. Mol Cell 2009, 35:586-597.

22. Stone M, Hartmann-Petersen $R$, Seeger M, Bech-Otschir D, Wallace M, Gordon C: Uch2/Uch37 is the major deubiquitinating enzyme associated with the $26 \mathrm{~S}$ proteasome in fission yeast. J Mol Biol 2004, 344:697-706.

23. Zhou L, Watts FZ: Nep1, a Schizosaccharomyces pombe deneddylating enzyme. Biochem J 2005, 389:307-314.

24. Mendoza HM, Shen LN, Botting C, Lewis A, Chen J, Ink B, Hay RT: NEDP1, a highly conserved cysteine protease that deNEDDylates Cullins. J Biol Chem 2003, 278:25637-25643.

25. Cope GA, Suh GS, Aravind L, Schwarz SE, Zipursky SL, Koonin EV, Deshaies RJ: Role of predicted metalloprotease motif of Jab1/Csn5 in cleavage of Nedd8 from Cul1. Science 2002, 298:608-611.

26. Mundt KE, Liu C, Carr AM: Deletion mutants in COP9/signalosome subunits in fission yeast Schizosaccharomyces pombe display distinct phenotypes. Mol Biol Cell 2002, 13:493-502.

27. Kouranti I, McLean JR, Feoktistova A, Liang P, Johnson AE, Roberts-Galbraith RH, Gould KL: A global census of fission yeast deubiquitinating enzyme localization and interaction networks reveals distinct compartmentalization profiles and overlapping functions in endocytosis and polarity. PLOS Biol 2010, 8:e1000471.

28. Reyes-Turcu FE, Ventii KH, Wilkinson KD: Regulation and cellular roles of ubiquitin-specific deubiquitinating enzymes. Annu Rev Biochem 2009, 78:363-397.

29. Hjerpe R, Thomas Y, Chen J, Zemla A, Curran S, Shpiro N, Dick LR, Kurz T: Changes in the ratio of free NEDD8 to ubiquitin triggers NEDDylation by ubiquitin enzymes. Biochem J 2012, 441:927-936.

30. Leidecker O, Matic I, Mahata B, Pion E, Xirodimas DP: The ubiquitin E1 enzyme Ube1 mediates NEDD8 activation under diverse stress conditions. Cell Cycle 2012, 11:1142-1150.

31. Singh RK, Zerath S, Kleifeld O, Scheffner M, Glickman MH, Fushman D: Recognition and Cleavage of Rub1 and Rub1-Ubiquitin Chains by Components of the Ubiquitin-Proteasome System. Mol Cell Proteomics 2012, 11:1595-1611.

doi:10.1186/1471-2091-14-8

Cite this article as: O'Donoghue et al.: Nedd8 processing enzymes in Schizosaccharomyces pombe. BMC Biochemistry 2013 14:8. 